\title{
$\beta$-Adrenergic receptors in the failing heart: the good, the bad, and the unknown
}

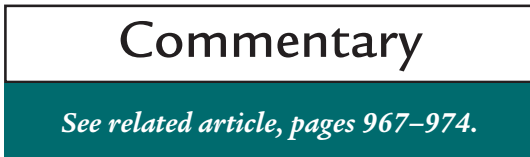

\author{
Stephen B. Liggett \\ Department of Medicine, University of Cincinnati College of Medicine, 231 Albert Sabin Way, Room G167, \\ Cincinnati, Ohio 45267-0564, USA. Phone: (513) 558-4831; Fax: (513) 558-0835; E-mail: stephen.liggett@uc.edu.
}

One of the most effective means of increasing cardiac output is by activating cardiomyocyte $\beta$-adrenergic receptors ( $\beta$ ARs). $\beta$ ARs couple primarily to the stimulatory $\mathrm{G}$-protein $\mathrm{G}_{\mathrm{s}}$, which activates adenylyl cyclase; increasing intracellular cAMP levels activate protein kinase A to phosphorylate its substrates troponin I, the L-type $\mathrm{Ca}^{2+}$-channels, and phospholamban (PLB), thus enhancing contractility. In the case of PLB, phosphorylation relieves its inhibition of the sarcoplasmic reticulum $\mathrm{Ca}^{2+}$-ATPase, thereby altering $\mathrm{Ca}^{2+}$ cycling (1). $\beta A R$ agonists, such as dobutamine, that rapidly increase contractility of the heart have become a mainstay in the acute treatment of decompensated heart failure.

This pathway has also been investigated for potential therapies to treat chronic heart failure, but here paradox abounds. In chronic human heart failure and in many animal models of the syndrome, $\beta$ AR function is, unexpectedly, limited by several molecular mechanisms. These include a decrease in the expression and coupling of the $\beta_{1} \mathrm{AR}$ subtype, a decrease in the coupling of the $\beta_{2}$ AR subtype, an increase in expression of the inhibitory $G$ protein $G_{i}$, an increase in the expression of the $\beta A R$ kinase (which phosphorylates and desensitizes $\beta$ ARs), and a decrease in expression or function of adenylyl cyclase. Because the consequent decrease in $\beta A R$ signaling limits energy expenditures in a heart that has little metabolic reserve, this response is generally thought to be adaptive. Indeed, judicious administration of $\beta A R$ antagonists ( $\beta$ blockers) in chronic heart failure can improve cardiac performance (2). However, because these changes each alter $\beta A R$ function in different ways, it would be naive to assume that they are all beneficial; some may well be adaptive - acting to oppose the progression of failure - while others are maladaptive. Delineating the mechanisms that uncouple $\beta$ AR activity from contractility in models of failing ventricular function should provide insight into the critical lesions for adaptive and maladaptive regulation and help identify the most appropriate targets for therapeutic intervention.

Toward this end, a number of transgenic and gene ablation mice have been created, in which various components of the pathway are amplified or missing. These studies have yielded some intriguing results. For example, a low level of $\beta_{2} \mathrm{AR}$ overexpression in the hearts of transgenic mice is well tolerated, with persistent enhancement of ejection fraction and absence of histopathological findings $(3,4)$. In contrast, low-level overexpression of the $\beta_{1}$ AR subtype results in cardiomyopathy with depressed contractile function $(5,6)$. Such results indicate that although $\beta_{1}$ AR and $\beta_{2}$ AR each couple to $G_{s}$, these receptor subtypes must engage distinct signaling pathways. Thus, it appears that $\beta_{2} \mathrm{AR}$, but not the $\beta_{1} A R$, can couple to the inhibitory G-protein, $G_{i}$, which may lead to an attenuated cAMP response (7) or to the activation of other, less-well-defined pathways (8). $\beta_{2}$ AR can also affect ion flow through the type III $\mathrm{Na}^{+} / \mathrm{H}^{+}$exchanger by binding the

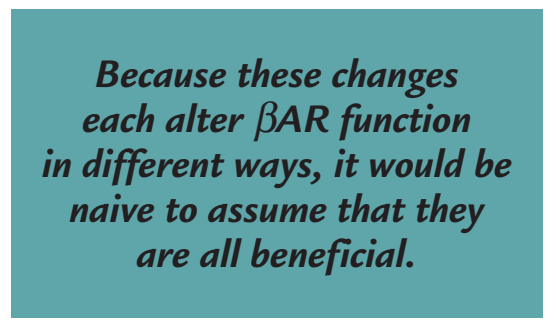

$\mathrm{Na}^{+} / \mathrm{H}^{+}$exchanger regulatory factor (9). Recent studies (10) also indicate that the two subtypes have different distributions within membrane microdomains of the myocyte, which may be another critical distinction. Interestingly, overexpression of adenylyl cyclase types V and VI in transgenic mouse hearts results in enhanced ventricular performance without apparent deleterious effects $(11,12)$, suggesting that cAMP alone may not be the only second messenger that is necessary for adrenergic mediated toxic effects. As the characterization of these genetically altered mice unfolds, various crossbreeding experiments are being carried out between mouse models of cardiomyopathy and other transgenic or knockout lines, in hopes of correcting specific aspects of the deranged signaling seen in the various models. In this issue of the JCI, Freeman et al. (13) report on the outcomes of altering three components of the signal transduction pathway in a mouse model of hypertrophic cardiomyopathy (HCM). HCM mice overexpress a modified myosin heavy chain, which results in hypertrophy followed by ventricular dilatation, depressed fractional shortening and exercise intolerance $(14,15)$. Freeman et al. (13) crossbred these mice, which show evidence of $\beta A R$ dysfunction, with other strains that either overexpress the $\beta_{2} \mathrm{AR}$ in the heart, express a $\beta A R$ kinase inhibitor ( $\beta A R K c t)$ in the heart, or are genetically ablated for PLB (PLB-null). Previous studies, confirmed here, showed that these perturbations each lead to enhanced contractility (16-18). However, their effects on the course of ventricular failure in the $\mathrm{HCM}$ mice were quite different. The $\mathrm{HCM} / \beta_{2} \mathrm{AR}$ mice initially show enhanced systolic function over that of nontransgenics, but by 8 months, the fractional shortening of their cardiac muscles is reduced, and half of the mice are dead. In contrast, HCM/PLB-null mice and HCM/ $\beta A R K c t$ mice show normal fractional shortening throughout the 12 -month study period. Furthermore, hypertrophy occurs in the $\mathrm{HCM} / \beta_{2} \mathrm{AR}$ and HCM/PLB-null mice but not the $\mathrm{HCM} / \beta \mathrm{ARK}$ ct mice. The expression profiles of three hypertrophy related genes, $\beta$-myosin heaving chain, atrial natriuretic factor, and $\alpha$-skeletal actin also differ in the groups. Normal expression of these genes is not found in any of the crossbred mice, but PLB-null-crossed mice showed the greatest improvement 
in $\beta$-myosin heavy chain expression, whereas the most favorable response for atrial natriuretic factor and $\alpha$-skeletal actin was with the $\beta$ ARKct-crossed mice. Given that the $\beta A R K c t$ mice also show no evidence of myocardial hypertrophy, as assessed by heart-to-body weight ratios, it appears that expression of this peptide had the most favorable chronic effects of the crosses, within the context of the HCM phenotype.

A consistent finding in these types of crossbreeding experiments with the $\beta A R K c t$ animal is that beneficial effects are only found when $\beta A R K$ levels or activities are increased. Thus, in the muscle-specific LIM knockout (19), the HCM mouse, and the calsequestrin overexpression (20) models, $\beta$ ARK is increased, and in each case, transgenic overexpression of the inhibitor peptide substantially improves function. In contrast, the $\mathrm{G}_{\alpha q}$ overexpressing model of cardiomyopathy, which also displays $\beta A R$ desensitization, hypertrophy, and marked ventricular dysfunction (21), does not have elevated $\beta A R K$ levels, and in these cases, ventricular function and $\beta A R$ responsiveness are not rescued with the $\beta$ ARK inhibitor $(22,23)$. Instead, restoration of other dysfunctional components of $\beta A R$ signaling improves function in vitro or in vivo (23-25).

The $\beta$ ARKct peptide acts by binding the $\beta \gamma$ subunits that are released from $\mathrm{G}$-protein heterotrimers. $\beta \gamma$ is required for $\beta A R K$ translocation and thus its ability to phosphorylate $\beta$ ARs. Since its beneficial effects are seen only in systems that feature elevated kinase activity, it appears that the inhibitory $\beta A R K c t$ peptide acts specifically on this aspect of the pathogenesis. However, other physiologic and biochemical indices of hypertrophy are also improved by $\beta A R K c t$, and it is intriguing to consider whether blocking $\beta \gamma$ may have effects other than inhibiting $\beta$ ARK. Indeed, $\beta \gamma$ stimulates tyrosine kinase signaling and phospholipase $C$ activation, which could accelerate hypertrophy. Another consideration is that as cardiac function improves when $\beta \gamma$ signaling is attenuated, $\beta$ AR function returns as a secondary response. This appears to be the case during $\beta$ blocker treatment, as $\beta$ AR function has been reported to improve during successful therapy (2). Finally, $\beta$ ARK phosphorylates multiple $G$ protein-coupled receptors, so the phenotypic improvement in animals expressing the inhibitory peptide may be explained in part by the ability of $\beta$ ARK to desensitize other receptors. It should be noted that $\beta_{2} \mathrm{AR}$ overexpression (23) and PLB ablation $(26,27)$ have improved a number of phenotypic characteristics of other models of cardiomyopathy. A coherent picture of how interdiction at these various points in the pathway can sometimes afford qualitatively similar rescue is still lacking.

Looking ahead to human therapy, because the strategy employed will surely depend on the etiology of the failure and the need for acute or chronic therapy, crossbreeding experiments like those of Freeman et al. (13) are crucial to identify strategies to pursue (or avoid) for the clinical modification of heart failure. However, understanding how these approaches achieve their effects will ultimately provide the greatest impetus for developing new genetic or pharmacologic therapies for human heart failure.

1. Brittsan, A.G., and Kranias, E.G. 2000. Phospholamban and cardiac contractile function. J. Mol. Cell. Cardiol. 32:2131-2139.

2. Bristow, M.R. 2000. Mechanistic and clinical rationales for using beta-blockers in heart failure. J. Card. Fail. 6:8-14.

3. Turki, J., Pak, J., Green, S., Martin, R., and Liggett, S.B. 1995. Genetic polymorphisms of the $\beta_{2}$ adrenergic receptor in nocturnal and non-nocturnal asthma: evidence that Gly16 correlates with the nocturnal phenotype. J. Clin. Invest 95:1635-1641.

4. Liggett, S.B., et al. 2000. Early and delayed consequences of $\beta_{2}$-adrenergic receptor overexpression in mouse hearts. Circulation. 101:1707-1714.

5. Engelhardt, S., Hein, L., Wiesmann, F., and Lohse, M.J. 1999. Progressive hypertrophy and heart failure in beta1-adrenergic receptor transgenic mice. Proc. Natl. Acad. Sci. USA. 96:7059-7064.

6. Bisognano, J.D., et al. 2000. Myocardial-directed overexpression of the human beta(1)-adrenergic receptor in transgenic mice. J. Mol. Cell. Cardiol. 32:817-830.

7. Tepe, N.M., and Liggett, S.B. 2000. Functional receptor coupling to $G_{i}$ is a mechanism of agonistpromoted desensitization of the $\beta_{2}$-adrenergic receptor. J Recept. Signal Transduct. Res. 20:75-85.

8. Daaka, Y., Luttrell, L.M., and Lefkowitz, R.J. 1997. Switching of the coupling of the $\beta_{2}$-adrenergic receptor to different $G$ proteins by protein kinase A. Nature. 390:88-91.

9. Hall, R.A., et al. 1998. The $\beta_{2}$-adrenergic receptor interacts with the $\mathrm{Na}^{+} / \mathrm{H}^{+}$-exchanger regulatory factor to control $\mathrm{Na}^{+} / \mathrm{H}^{+}$exchange. Nature. 392:626-630.

10. Rybin, V.O., Xu, X., Lisanti, M.P., and Steinberg,
S.F. 2000. Differential targeting of beta-adrenergic receptor subtypes and adenylyl cyclase to cardiomyocyte caveolae. A mechanism to functionally regulate the CAMP signaling pathway. J. Biol. Chem. 275:41447-41457.

11. Tepe, N.M., et al. 1999. Altering the receptoreffector ratio by transgenic overexpression of type $V$ adenylyl cyclase: enhanced basal catalytic activity and function without increased cardiomyocyte $\beta$-adrenergic signalling. Biochemistry. 38:16706-16713.

12. Gao, M.H., et al. 1999. Adenylylcyclase increases responsiveness to catecholamine stimulation in transgenic mice. Circulation. 99:1618-1622.

13. Freeman, K., et al. 2001. Alterations in cardiac adrenergic signaling and calcium cycling differentially affect the progression of cardiomyopathy. J. Clin. Invest. 107:967-974.

14. Vikstrom, K.L., Factor, S.M., and Leinwand, L.A. 1996. Mice expressing mutant myosin heavy chains are a model for familial hypertrophic cardiomyopathy. Mol. Med. 2:556-567.

15. Freeman, K., et al. 2001. Progression from hypertrophic to dilated cardiomyopathy in mice that express a mutant myosin transgene. Am. J. Physiol. Heart Circ. Physiol. 280:H151-H159.

16. Milano, C.A., et al. 1994. Enhanced myocardial function in transgenic mice overexpressing the $\beta_{2}$-adrenergic receptor. Science. 264:582-586.

17. Koch, W.J., et al. 1995. Cardiac function in mice overexpressing the $\beta$-adrenergic receptor kinase or a $\beta A R K$ inhibitor. Science. 268:1350-1353.

18. Luo, W., et al. 1994. Targeted ablation of the phospholamban gene is associated with markedly enhanced myocardial contractility and loss of $\beta$-agonist stimulation. Circ. Res. 75:401-409.

19. Rockman, H.A., et al. 1998. Expression of a betaadrenergic receptor kinase 1 inhibitor prevents the development of myocardial failure in gene-targeted mice. Proc. Natl. Acad. Sci. USA. 95:7000-7005.

20. Harding, V., Jones, L., Lefkowitz, R.J., Koch, W.J., and Rockman, H.A. 2001. Cardiac BARK1 inhibition prolongs survival and augments $\beta$ blocker therapy in a mouse model of severe heart failure. Proc. Natl. Acad. Sci. USA. In press.

21. D’Angelo, D.D., et al. 1997. Transgenic G $\alpha$ q overexpression induces cardiac contractile failure in mice. Proc. Natl. Acad. Sci. USA. 94:8121-8126.

22. Dorn, G.W.I., Tepe, N.M., Wu, G., Yatani, A., and Liggett, S.B. 2000. Mechanisms of impaired $\beta$ adrenergic receptor signaling in $\mathrm{G}_{\alpha q}$-mediated cardiac hypertrophy and ventricular dysfunction. Mol. Pharmacol. 57:278-287.

23. Dorn, G.W., II., Tepe, N.M., Lorenz, J.N., Koch, W.J., and Liggett, S.B. 1999. Low- and high-level transgenic expression of $\beta_{2}$-adrenergic receptors differentially affect cardiac hypertrophy and function in $\mathrm{G}_{\alpha q^{-}}$-overexpressing mice. Proc. Natl. Acad. Sci. USA. 96:6400-6405.

24. Tepe, N.M., and Liggett, S.B. 1999. Transgenic replacement of type $V$ adenylyl cyclase identifies a critical mechanism of $\beta$-adrenergic receptor dysfunction in the $\mathrm{G}_{\alpha q}$ overexpressing mouse. FEBS Lett. 458:236-240.

25. Roth, D.M., et al. 1999. Cardiac-directed adenylyl cyclase expression improves heart function in murine cardiomyopathy. Circulation. 99:3099-3102.

26. Minamisawa, S., et al. 1999. Chronic phospholamban-sarcoplasmic reticulum calcium ATPase interaction is the critical calcium cycling defect in dilated cardiomyopathy. Cell. 99:313-322.

27. Sato, Y., et al. 2001. Rescue of contractile param eters and myocyte hypertrophy in calsequestrin overexpressing myocardium by phospholamban ablation. J. Biol. Chem. In press. 